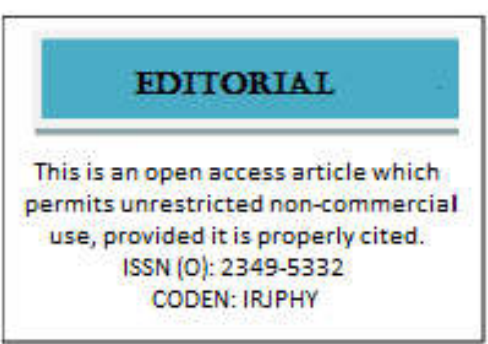

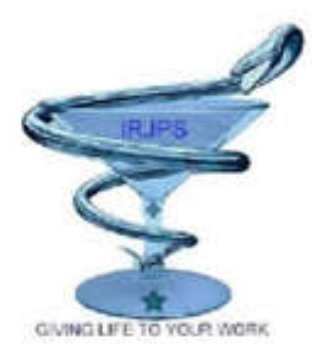

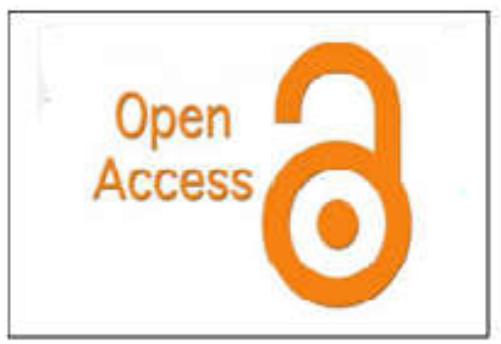

\title{
EDITORIAL
}

\section{MOVING TOWARDS A NEW NORMAL}

As we are moving towards a new normal to coexist with COVID19, here are a few steps offices and institutions can adopt while reopening after the worldwide lockdown, which also should be on display:

1. Use Mask and Gloves on a regular basis and keep on replacing them at regular intervals.

2. Sanitize your hands after you touch surfaces while transportation or at office.

3. Maintain social distancing everywhere.

4. Don't touch your face, mouth or nose, as this increases the risk of infection.

5. Make sure, you do not sneeze or cough in open.

6. Carry your stationery from home to reduce the risk of being contaminated through office stationery.

7. Avoid outside food and go for home cooked food.

8. If you grow any COVID19 symptom like; cold, fever, breathlessness or decreased sense of taste or smell, you should stop going to the office and ask for medical health.

9. Avail all possible proactive service and maintenance programmes from here on.

10. Adhere to guidelines on hygiene practices by the government, maintaining social distancing and limiting personnel interaction.

11. Offices and institutions should setup protocols for screening at premise via infra-red thermometers.

12. A daily update status on COVID19 status should be displayed.

13. Ensure basic soft-hygiene services at workplace.

14. A well articulated cleaning SOP with specifications should be in place.

15. Installing of touch less taps and soap dispensers is need of the hour.

16. Keeping a track of employees and visitors along with a look at visible symptoms.

17. Sanitizing hands after each cash transaction should be ensured.

18. For Official tours and travels; travel advisory of the local authorities are to be observed.

Prof. (Dr.) Saumendu Deb Roy

Principal,

Mata Gujri College of Pharmacy

Kishanganj, Bihar.
Indian Research Journal of Pharmacy and Science; 25(2020)2112;

Journal Home Page: https://www.irjps.in

DOI: $10.21276 /$ irjps.2020.7.2.1 